\title{
Redução de riscos de desastres na produção sobre educação ambiental: um panorama das pesquisas no Brasil
}

\author{
Disaster risk reduction in environmental education production: an overview of research \\ in Brazil
}

\section{Reducción del riesgo de desastres en la producción de educación ambiental: una visión general de la investigación en Brasil}

\author{
Patricia Mie Matsuo ${ }^{1}$ \\ Sandra Aparecida de Oliveira e Souza ${ }^{2}$ \\ Rosana Louro Ferreira Silva ${ }^{3}$ \\ Rachel Trajber ${ }^{4}$
}

\begin{abstract}
Resumo
Este trabalho teve como objetivo analisar a temática redução de riscos de desastres (RRD) na produção acadêmica de educação ambiental no Brasil, no período de 1981-2018, identificando suas características, lacunas e oportunidades. A busca foi realizada com seis descritores nos títulos e resumos de seis plataformas. Os 26 estudos analisados apresentaram múltiplas abordagens e onze categorias temáticas foram estabelecidas a partir da análise de conteúdo. A maioria dos trabalhos estava relacionada à percepção, ao ensino e currículo e à gestão e políticas públicas. As principais lacunas foram encontradas no reduzido número de pesquisas sobre formação, participação, comunicação e fatores geradores de risco. Apenas um trabalho utilizou o termo RRD e nenhum trabalho relacionado às secas foi identificado. Os resultados subsidiam futuros caminhos da produção acadêmica e evidenciam a necessidade de investigação e aprofundamento da RRD no campo da educação ambiental.
\end{abstract}

Palavras-chave: Educação ambiental. Redução de riscos de desastres. Riscos. Desastres. Estado da arte.

\begin{abstract}
This study aimed to analyze the theme disaster risk reduction (DRR) in the academic production of environmental education in Brazil from 1981-2018, identifying its characteristics, gaps and opportunities. The search was conducted with six descriptors in the titles and abstracts in six platforms. The 26 studies analyzed presented multiple approaches and 11 thematic categories were established from content analysis. Most of the publications was related to perception, teaching and curriculum, and to public policy and management. The main gaps were found in the small number of research on training and stakeholders participation, communication and risk factors. Only one paper used the term DRR and any work related to drought was identified. The results subsidize future paths of academic production and highlight the need for research and deepening in the field of environmental education aligned with DRR.
\end{abstract}

Keywords: Environmental education. Disaster risk reduction. Risks. Disasters. State of art.

\section{Resumen}

\footnotetext{
${ }^{1}$ Doutoranda do Programa de Pós-Graduação Interunidades em Ensino de Ciências. Universidade de São Paulo. Bolsista CNPq. E-mail: patricia.matsuo@usp.br

${ }^{2}$ Mestra em Educação, Arte e História da Cultura pela Universidade Presbiteriana Mackenzie. Professora de Ensino Fundamental (Ciências) da Secretaria da Educação do Estado de São Paulo. E-mail: sanvla@uol.com.br

${ }^{3}$ Doutora em Educação pela Universidade de São Paulo. Professora do Departamento de Zoologia do Instituto de Biociências, Universidade de São Paulo. Orientadora do Programa de Pós-Graduação Interunidades em Ensino de Ciências. E-mail: rosanas@usp.br

4 Doutora em Antropologia pela Purdue University/revalidação na Universidade Federal de São Carlos. Coordenadora do Programa Cemaden Educação do Centro Nacional de Monitoramento e Alerta de Desastres Naturais (Cemaden). Bolsista CNPq. E-mail: rachel.trajber@cemaden.gov.br
} 
Este trabajo tuvo como objetivo analizar el tema de la reducción de riesgos de desastres (RRD) en la producción académica de educación ambiental en Brasil en el período 1981-2018, identificando sus características, deficiencias y oportunidades. La búsqueda fue realizada con seis descriptores en los títulos y resúmenes de seis plataformas. Los 26 estudios analizados presentaron múltiples enfoques y 11 categorías temáticas se establecieron a partir del análisis de contenido. La mayoría de los trabajos estaban relacionados con percepción, enseñanza y currículo, y gestión y políticas públicas. Las principales deficiencias fueron encontradas en las reducidas investigaciones sobre formación y participación, comunicación y factores generadores de riesgo. Sólo un trabajo utilizó el término RRD y ningún trabajo con las sequías fue identificado. Los resultados subsidian futuros caminos de la producción académica y evidencian la necesidad de investigación y profundización en el campo de la educación ambiental alineado a la RRD.

Palabras clave: Educación ambiental. Reducción de riesgos de desastres. Riesgos. Desastres. Estado del arte.

\section{Introdução}

O conceito de sociedade de risco foi apresentado, em 1986, pelo sociólogo alemão Ulrich Beck, como a sociedade no qual a produção social de riqueza é acompanhada pela produção social de risco. Esses riscos, quando gerados, são compartilhados com todas as classes sociais (BECK, 2010).

Para o Escritório das Nações Unidas para a Redução do Risco de Desastres (United Nations Office for Disasters Risk Reduction) - UNISDR (2009), risco é a combinação da probabilidade de um evento ocorrer e suas consequências negativas. Segundo Marchezini et al. (2017a, p. 45) o termo risco pode ser compreendido como uma função da:

[...] (1) exposição a uma ameaça, (2) suscetibilidade ou sensibilidade a danos ou perdas, (3) grau de proteção pessoal ou social e (4) capacidade para lidar ou se adaptar ao impacto da ameaça [....] geralmente é entendido como a interação entre uma população vulnerável e as ameaças naturais, biológicas e/ou tecnológicas.

Trajber, Olivato e Marchezini (2015) complementam que risco é a chance de que a população e seus bens materiais sofram consequências prejudiciais ou perdas diante do impacto de ameaças naturais ou tecnológicas.

Já desastre é definido como o resultado da combinação de ameaças, condições de vulnerabilidade e da insuficiente capacidade para reduzir as consequências negativas. Os impactos incluem mortes, doenças, danos econômicos, degradação ambiental, perda de serviços e problemas sociais (UNISDR, 2009).

Para Wisner et al. (2003) desastres são uma construção social, criados a partir de um conjunto de processos naturais com ações humanas, relações sociais, políticas e econômicas. Nesse sentido, a temática demanda uma abordagem integrada que se alinha à Teoria da Complexidade, com a compreensão de que o pensamento complexo é animado pelo saber não fragmentado e o reconhecimento do inacabado (MORIN, 2015). Ainda, segundo Jacobi (2014, p. 65) a "abordagem da complexidade coloca-se como uma alternativa para a busca de novas formas de gerar conhecimento, e promove uma inflexão na estrutura consolidada que gerou uma hierarquia de saberes".

As palavras riscos e desastres surgem associadas em uma nova área de conhecimento - redução de riscos de desastres (RRD), definida como:

[...] conceito e a prática de reduzir o risco de desastre por meio de esforços sistemáticos voltados à análise e gestão dos fatores geradores de desastres, incluindo a redução do grau de exposição a ameaças, redução da vulnerabilidade da população e propriedade, gestão do uso e ocupação do solo e melhoria da preparação para eventos adversos (UNISDR, 2009, p. 27, tradução nossa).

O Brasil começa a ter um aumento das ocorrências de desastres a partir de 1960 (TOMINAGA; SANTORO; AMARAL, 2015). Comparando dados dos desastres ocorridos nos 
últimos vinte anos (1999-2008) e (2009-2018) é possível identificar que o país segue uma tendência mundial, com aumento de $6,1 \%$ no número de eventos; $611,5 \%$ no número de pessoas atingidas, chegando a mais de 37 milhões de brasileiros; e os prejuízos econômicos passaram de US\$3,6 para US\$10,3 bilhões, segundo informe EM-DAT, do International Disaster Databases (2019).

Estudo recente do Instituto Brasileiro de Geografia e Estatística - IBGE, juntamente com o Centro Nacional de Monitoramento e Alertas de Desastres Naturais - CEMADEN (2018) indica que mais de 8,2 milhões de brasileiros estavam expostos aos riscos de desastres de origem hidrometeorológica em 872 municípios avaliados em todas as regiões, sendo 9,8\% da população na Região Sudeste, 11,4\% no Nordeste, 6,0\% no Sul, 3,9\% no Norte e o CentroOeste com $0,3 \%$. Esses números demonstram que a discussão sobre essa questão é imprescindível e urgente, já que tais fenômenos geram impactos significativos na área da saúde, urbanização, ambiental, econômica, educação e agricultura.

A educação é reconhecida como componente essencial para se criar uma cultura de prevenção de desastres e resiliência, configurando prioridades em documentos de referência sobre RRD da Organização das Nações Unidas, como o Marco de Ação de Hyogo 2005-2015 (UNICEF; UNISDR, 2011) e o Marco de Sendai para a Redução do Risco de Desastres 20152030 (UNISDR, 2015a).

No contexto da RRD, a educação pode, entre outros, contribuir para que as pessoas entendam as interações da sociedade com seus espaços de interlocução e no envolvimento dos processos participativos de uso e ocupação do solo. Da Silva-Rosa et al. (2015) e Sulaiman (2018) reforçam o papel da educação nos processos de compreensão das ameaças, mobilização da comunidade e construção de mudanças na forma de pensar e lidar com os riscos.

A educação e RRD podem ter uma abordagem simbiótica, ou seja, podem ser absorvidas por outras áreas com as quais tenham afinidade. Na América Latina, onde existe um forte histórico em educação ambiental, há um crescente reconhecimento de que a educação sobre a gestão de risco é uma aplicação específica da educação ambiental (SELBY; KAGAWA, 2012).

Sob essa perspectiva, a interação entre a educação e RRD se alinha com os princípios da educação ambiental crítica, com suas raízes nos ideais democráticos e emancipatórios, buscando a formação de grupos sociais capazes de identificar, problematizar e agir em relação às questões socioambientais (CARVALHO, 2004) e também, estabelece processos participativo-reflexivos que promovem a consolidação de uma sociedade sustentável com valores favoráveis à justiça social, fortalecendo o sentido de responsabilidade cidadã e de pertencimento local, partindo de pressupostos não apenas teóricos, mas também políticos, éticos e ideológicos (LOUREIRO, 2004; SILVA; CAMPINA 2011).

No Brasil, a urgência em se tratar desse tema, no contexto educativo, não se deve apenas às previsões de aumento de ocorrência de desastres naturais por causa das mudanças climáticas (MARENGO, 2010; IPCC, 2014; COUTINHO; RAMOS, 2018), mas, também, pelo fato de existirem em todas as regiões do país, escolas localizadas em áreas de risco. A pesquisa de Marchezini, Muñoz e Trajber (2018a) identificou 2.438 escolas em áreas de risco hidrológico e/ou geológico. Desse total, 74\% são escolas públicas e mais de três milhões de pessoas estão vinculadas a essas áreas.

Por se tratar de uma temática relativamente recente no Brasil, o objetivo deste trabalho foi analisar a produção acadêmica no campo da educação ambiental relacionada à RRD em seis plataformas, identificando suas características, lacunas e oportunidades nesse novo campo de conhecimento 


\section{Procedimentos metodológicos}

O procedimento metodológico utilizado foi a pesquisa denominada estado da arte, que apresenta caráter bibliográfico, inventariante e descritivo, e tem a perspectiva de mapear e discutir uma produção acadêmica nos variados campos do conhecimento, procurando responder às questões discutidas e destacadas em diferentes épocas e lugares e de que modo têm sido produzidas determinadas dissertações, teses, publicações em periódicos e comunicações em anais de seminários e de congressos (FERREIRA, 2002).

Nessa perspectiva, Romanowski e Ens (2006) esclarecem que o estado da arte pode contribuir com a teoria e a prática de uma área de conhecimento, pois procura apontar limitações sobre o campo em que se move a pesquisa, bem como as lacunas presentes e, também, pode identificar experiências inovadoras.

Iniciamos com a definição dos descritores de busca. Além de redução de riscos de desastres, estabelecemos outros cinco termos gerados pelas variações das palavras riscos e desastres: desastres ambientais, desastres naturais, desastres socioambientais, riscos ambientais e riscos socioambientais. Os descritores foram utilizados em buscas nos títulos e nos resumos de seis bancos de dados do período de 1981 a 2018. As bases de consulta foram selecionadas com o intuito de trazer a variedade de representações da produção de pesquisa de educação ambiental e incluíram anais de eventos, repositórios de teses e dissertações e alguns dos periódicos de referência na área em questão:

1) Encontro Pesquisa em Educação Ambiental (EPEA): anais do encontro bianual iniciado em 2001 (www.epea.tmp.br).

2) Pesquisa em Educação Ambiental: periódico semestral iniciado em 2006 (www.periodicos.rc.biblioteca.unesp.br/index.php/pesquisa).

3) Projeto Estado da Arte da Pesquisa em Educação Ambiental (EArte): banco de teses e dissertações concluídas no período de 1981 a 2016 (www.earte.net/).

4) Revista Ambiente e Sociedade: periódico trimestral publicado desde 1997 (www. anppas.org.br/novosite/index.php? $\mathrm{p}=\mathrm{aes}$ ). As buscas dos termos foram associadas à palavra educação.

5) Revista Brasileira de Educação Ambiental (RevBEA): periódico semestral publicado desde 2004 (www.periodicos.unifesp.br/index.php/revbea).

6) Revista Eletrônica do Mestrado em Educação Ambiental(REMEA): periódico quadrimestral publicado desde 1999 (www.periodicos.furg.br/remea).

O levantamento foi realizado até março de 2019 e foram encontrados 45 trabalhos. A primeira etapa desse trabalho constituiu-se em uma leitura preliminar dos títulos e resumos, com a finalidade de buscar pesquisas em educação ambiental que apresentassem em seu contexto relações com um dos descritores. O procedimento adotado resultou na retirada de dezenove pesquisas, a maioria por não apresentar foco nos termos pesquisados, e um caso em que o mesmo resumo foi publicado em duas das bases analisadas (Figura 1).

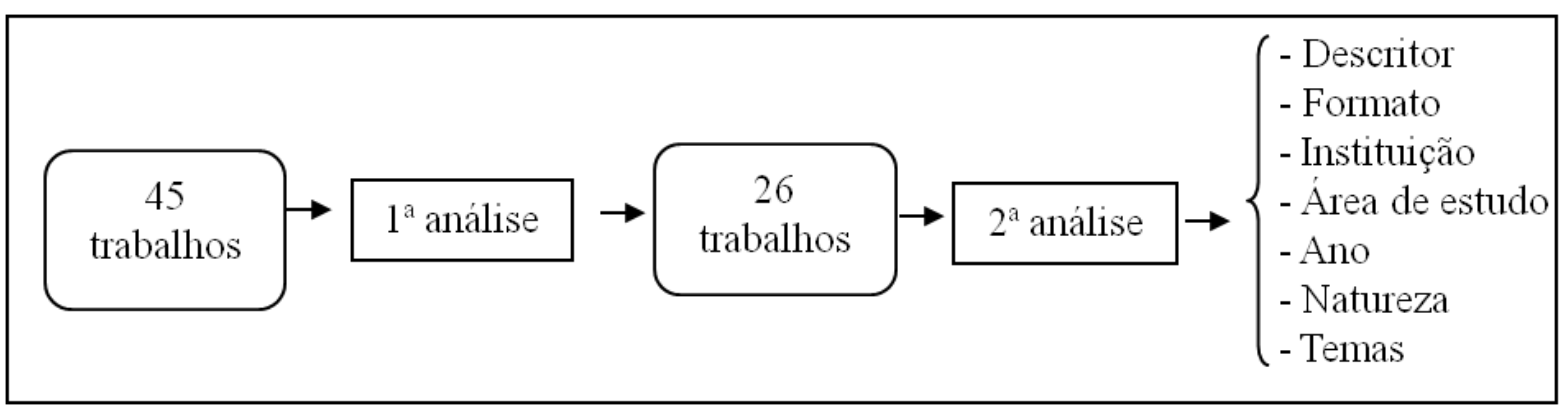

Figura 1 - Fluxo do procedimento metodológico 
Fonte: elaborada pelas autoras

A segunda etapa ocorreu de forma qualitativa e quantitativa e buscou caracterizar as 26 pesquisas que atenderam à proposta desse estado da arte, identificando a frequência dos descritores, o formato, a representatividade institucional, áreas de estudo, o período de publicação das pesquisas, a natureza dos riscos/desastres abordados e os principais temas. Foram construídas categorias temáticas a posteriori, utilizando contribuições da análise de conteúdo (BARDIN, 2016; FRANCO, 2003).

\section{Panorama da temática RRD nas pesquisas em educação ambiental}

Os 26 trabalhos encontrados representam $0,37 \%$ do total da produção acadêmica considerada neste estudo. A REMEA foi a que apresentou o maior valor com $0,69 \%$ do total de trabalhos publicados no periódico, com seis artigos (Tabela 1).

Tabela 1 - Quantidade de publicações encontradas em cada base de consulta até março de $2019(\mathrm{~N}=26)$

\begin{tabular}{c|c|c|c|c|c|c|c}
\hline Fontes & EPEA & $\begin{array}{c}\text { Pesquisa } \\
\text { em EA }\end{array}$ & EArte & $\begin{array}{c}\text { Ambiente e } \\
\text { Sociedade }\end{array}$ & RevBEA & REMEA & Total \\
\hline Total de publicações encontradas & 3 & 1 & 14 & 1 & 1 & 6 & 26 \\
\hline Total de publicações na base & 837 & 245 & 4.205 & 483 & 374 & 869 & 7.013 \\
\hline
\end{tabular}

O Projeto EArte apresentou o maior número de publicações (14) e a maior diversidade, com cinco dos seis descritores pesquisados (Tabela 2), fato já esperado por conter o maior número de documentos, entretanto esse valor representou apenas $0,33 \%$ de toda produção acadêmica no Earte.

Tabela 2 - Quantidade de publicações encontradas para cada descritor, nas seis bases de consulta, até março de 2019

\begin{tabular}{l|c|c|c|c|c|c|c}
\hline \multicolumn{1}{c|}{ Fontes } & \multirow{2}{c}{ EPEA } & $\begin{array}{c}\text { Pesquisa } \\
\text { em EA }\end{array}$ & EArte & $\begin{array}{c}\text { Ambiente e } \\
\text { Sociedade }\end{array}$ & RevBEA & REMEA & $\begin{array}{c}\text { Qtde. de } \\
\text { publicações }\end{array}$ \\
\cline { 1 - 7 } Redução de Riscos de Desastres & 0 & 0 & 0 & $1 *$ & 0 & 0 & 1 \\
\hline Desastres Ambientais & 1 & 0 & 2 & 0 & 0 & $4^{*}$ & 7 \\
\hline Desastres Naturais & 0 & 1 & 3 & 0 & 1 & 0 & 5 \\
\hline Desastres Socioambientais & 0 & 0 & 1 & 0 & 0 & 0 & 1 \\
\hline Riscos Ambientais & 1 & 0 & 7 & 0 & 0 & $4^{*}$ & 12 \\
\hline Riscos Socioambientais & 1 & 0 & 1 & $1 *$ & 0 & 0 & 3 \\
\hline
\end{tabular}

* Três trabalhos apresentam mais de um descritor.

$$
\text { Fonte: elaborada pelas autoras }
$$

O descritor mais frequente foi riscos ambientais, abordado em $46 \%$ das publicações consideradas, seguidos de desastres ambientais (27\%), desastres naturais (19\%) e riscos socioambientais (12\%). Tanto o termo desastres socioambientais como redução de riscos de desastres aparecem em apenas uma publicação. Três trabalhos apresentaram mais de um dos termos pesquisados. Essa maior frequência observada do descritor riscos ambientais pode estar 
relacionada com o que Carpi Jr (2012, p. 33) relata que o "risco ambiental torna-se um termo sintético que abriga os demais sem que eles sejam esquecidos ou menosprezados".

Quanto ao formato de trabalhos, foram identificadas dissertações, teses, artigos em periódicos e comunicações em anais de eventos científicos. Considerando as diferenças quanto à natureza e características dos documentos, apenas os títulos e resumos das produções foram analisados, recebendo, assim, o mesmo peso neste trabalho. Dissertações tiveram a maior representatividade, com doze trabalhos $(46,2 \%)$, seguidos de artigos, com nove publicações $(34,6 \%)$. Comunicações em eventos foram representados com três resumos $(11,5 \%)$ e apenas duas teses $(7,7 \%)$.

No que diz respeito à representatividade institucional, as publicações foram analisadas da seguinte maneira: nas produções no formato de dissertações e teses foi considerada a instituição do programa de mestrado ou doutorado e, no caso dos artigos, a instituição de vínculo do(a) primeiro(a) autor(a). Foram identificadas 21 instituições diferentes, a maioria $(80,8 \%)$ foi produzida nas instituições públicas, sendo $57,7 \%$ da esfera federal, $11,5 \%$ estadual e 11,5\% municipal e 19,2\% nas particulares. Essas instituições estão localizadas em dez estados e no Distrito Federal, mais da metade $(57,7 \%)$ está localizada na região Sudeste, sendo Rio de Janeiro o estado com a maior representação neste estudo, com seis publicações, seguido por São Paulo e Santa Catarina, com quatro trabalhos cada e, na sequência, o estado de Minas Gerais com três trabalhos.

Outro aspecto observado foi a área de estudo dessas investigações. Foi verificada a realização de pesquisas nas regiões Sudeste, Sul, Nordeste e Centro-Oeste. Novamente, a região Sudeste foi a mais estudada, com nove pesquisas. Essa concentração de pesquisas no Sudeste também foi encontrada em estudos similares sobre a produção científica em educação ambiental no Brasil (MAZZUCATO; BACCI, 2017; CARVALHO; FARIAS, 2011). Apesar da região Norte não ter sido identificada como área de estudo nas pesquisas analisadas, acreditamos que isso não representa, necessariamente, que investigações de educação e RRD não estejam em andamento nessa região ou não tenham sido publicadas. Em dez trabalhos não foi possível identificar a área de estudo, entretanto verificou-se que se tratam em sua maioria de pesquisas teóricas ou de contextos mais amplos.

\subsection{As produções ao longo do tempo}

A análise temporal realizada levou em consideração os anos das publicações, o que não reflete diretamente o período em que as investigações foram desenvolvidas.

A primeira pesquisa que atendeu à proposta deste trabalho de estado de arte só foi publicada em 1994, apesar da ocorrência de eventos como o caso da poluição atmosférica em Cubatão/SP e de inundações e deslizamentos em Blumenau/SC (VIEIRA; JANSEN, 2018) na década de 1980 e do Brasil sediar a Conferência das Nações Unidas sobre o Meio Ambiente e o Desenvolvimento em 1992 (Tabela 3).

No período de 1995 a 2002 houve uma lacuna de publicações, podendo suscitar que a influência dos riscos e desastres nessas pesquisas tem início nas décadas seguintes, juntamente com o desenvolvimento da educação ambiental do Brasil. 
Tabela 3 - Número de publicações encontrado por ano, no período de 1994 a 2017 (N = 26)

\begin{tabular}{c|c}
\hline Ano* & Número de publicações \\
\hline 1994 & 1 \\
\hline 2003 & 1 \\
\hline 2005 & 1 \\
\hline 2009 & 1 \\
\hline 2010 & 1 \\
\hline 2011 & 3 \\
\hline 2012 & 3 \\
\hline 2013 & 3 \\
\hline 2014 & 1 \\
\hline 2015 & 5 \\
\hline 2016 & 3 \\
\hline 2017 & 3 \\
\hline TOTAL & $\mathbf{2 6}$ \\
\hline * Os anos sem publicação não foram incluídos na tabela. \\
\hline \multicolumn{2}{|c}{ Fonte: elaborada pelas autoras }
\end{tabular}

A incidência de publicações observada nos últimos anos pode ter sido influenciada por alguns fatores e acontecimentos no Brasil: 1) aumento da ocorrência de desastres, 2) repercussão pela mídia dos grandes desastres como os que ocorreram em Blumenau/SC, em 2008, Angra dos Reis/RJ, em 2009/2011, na região Serrana no Rio de Janeiro, em 2011 (MOURA, 2018); 3) criação do Centro Nacional de Monitoramento e Alertas de Desastres Naturais (CEMADEN) pelo Ministério de Ciência, Tecnologia e Inovação, em 2011; 4) criação da Lei No 12.608 - Política Nacional de Proteção e Defesa Civil (BRASIL, 2012); 5) aumento de $25 \%$ no número de programas de pós-graduação, no Brasil, entre os anos de 2013 e 2016 (CAPES, 2017) e 6) criação da Associação Brasileira de Pesquisa Científica, Tecnológica e Inovação em Redução de Riscos e Desastres, em 2013.

Nesse cenário de eventos, mobilização e institucionalização da temática em tela nas instâncias políticas e acadêmicas, espera-se que o próprio campo de conhecimento venha a crescer em diversos segmentos das pesquisas, incluindo, também, o do campo da educação ambiental.

\subsection{A natureza dos riscos e dos desastres}

Ao analisarmos a natureza dos riscos/desastres associados aos descritores, 50\% dos trabalhos não a identificaram e optamos em agrupá-los em riscos e desastres em geral. Dentre as publicações que a especificaram, quatro trabalhos mencionaram riscos/desastres de natureza hidrológica e geológica simultaneamente (15\%); três trabalhos $(11 \%)$ trataram apenas de eventos hidrológicos (inundações, enxurradas) e dois (8\%) abordaram apenas eventos de natureza geológica (movimento de massa, deslizamento de terra) (Figura 2).

Os riscos/desastres tecnológicos (dutoviários, pólo industrial, setor siderúrgico) foram identificados em três trabalhos (12\%). Saneamento apareceu em apenas um trabalho (4\%) e estava ligado aos riscos provenientes da falta de saneamento em um conjunto habitacional em Anápolis/GO.

Considerando as pesquisas que identificaram a natureza dos riscos/desastres, pudemos constatar que os eventos hidrológicos foram os mais estudados, o que coincide com os resultados de outra pesquisa de natureza estado da arte que analisou a ciência cidadã e gestão 
de desastres em 408 artigos científicos, publicados nos nove principais jornais de riscos e desastres, entre 2005 e junho de 2018 (MARCHEZINI et al.,2018b), na qual as inundações predominaram como principal ameaça.

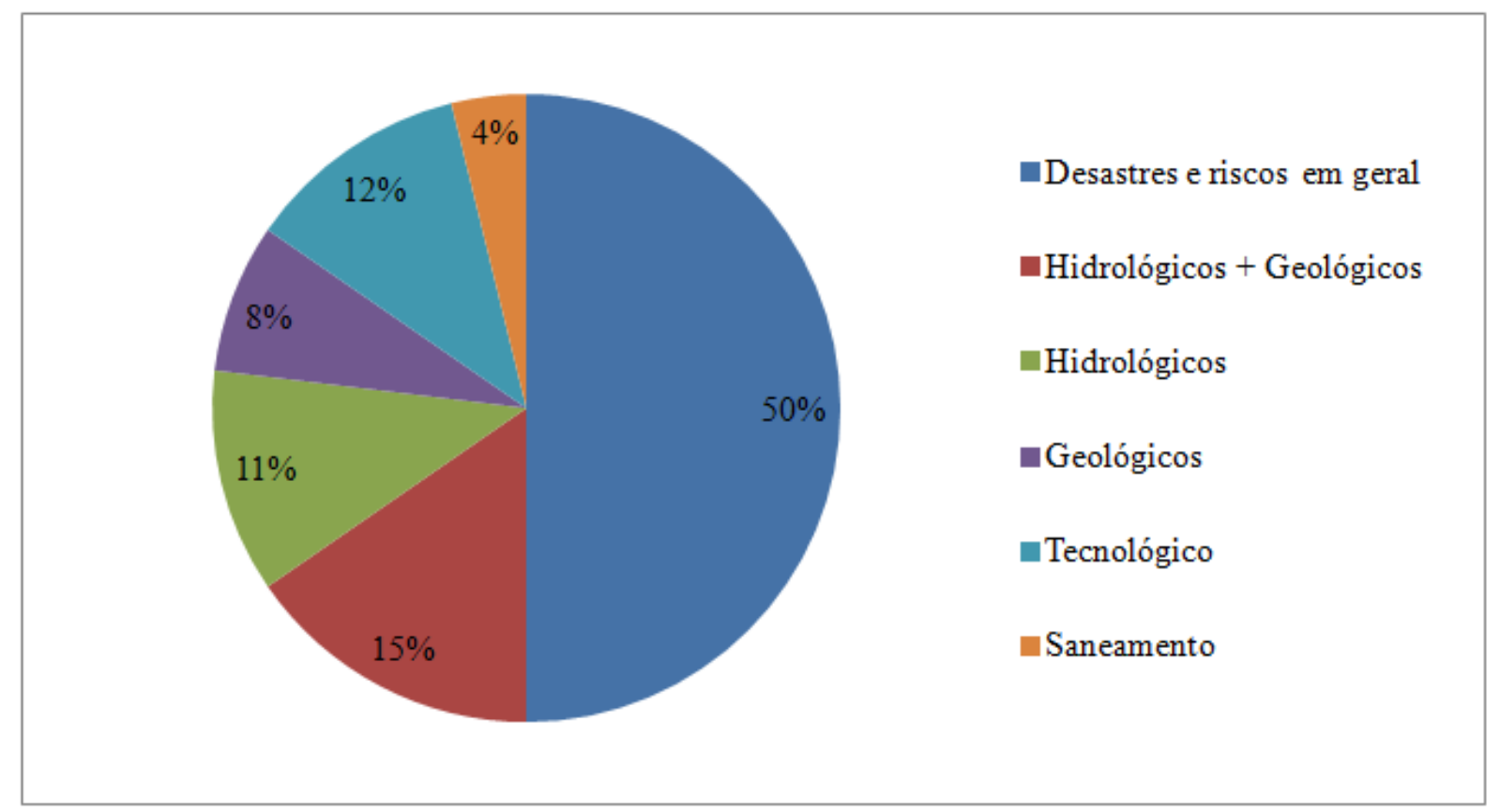

Figura 2 - Natureza dos riscos/desastres tratada nos trabalhos encontrados nas seis plataformas consultadas até março de $2019(\mathrm{~N}=30)$

Fonte: elaborada pelas autoras

Secas não foram abordadas em nenhum dos trabalhos considerados na presente pesquisa. O que pode indicar a necessidade de promover pesquisas envolvendo a problemática das secas, estiagens e déficit hídrico em investigações no campo da educação ambiental e RRD, pois dados do Perfil dos Municípios Brasileiros (IBGE, 2017) mostram que, entre 2013 e 2017, $48,6 \%$ dos municípios brasileiros foram afetados por secas. Além disso, as secas impactam negativamente tanto as populações vulneráveis, como também grandes áreas e podem perdurar por tempo prolongado (CUNHA et al., 2019).

\subsection{As pesquisas focam em...}

Foram encontradas múltiplas abordagens nas investigações, desde estudo mais teóricos com reflexões sobre o consumo, individualismo crescente; passando por análises específicas dos riscos ambientais como fator de risco de crédito nas instituições bancárias e os estudos das ciências da saúde sobre aspectos psicológicos dos atores sociais; até processos avaliativos de programas e projetos de educação ambiental de governo, empresas, escolas.

A identificação dos principais temas em que os descritores foram abordados foi realizada de acordo com os títulos e objetivos das pesquisas, conforme explicitados nos resumos. Identificamos uma diversidade de 24 temas, sendo que 61,5\% dos trabalhos abordaram dois ou mais temas. Os temas foram reorganizados em onze categorias temáticas $a$ posteriori (Quadro 1). 


\begin{tabular}{|l|l|c|}
\hline \multicolumn{1}{|c|}{\begin{tabular}{c}
\multicolumn{1}{|c|}{ Categorias } \\
temáticas
\end{tabular}} & \multicolumn{1}{|c|}{ Descrição } & $\begin{array}{c}\text { No de } \\
\text { trabalhos }\end{array}$ \\
\hline Percepção & $\begin{array}{l}\text { Percepção de riscos de atores sociais afetados ou não por } \\
\text { desastres }\end{array}$ & 7 \\
\hline Ensino e currículo & $\begin{array}{l}\text { Abordagens no ambiente escolar, avaliação de programas e } \\
\text { projetos, integração da temática no currículo }\end{array}$ & 7 \\
\hline $\begin{array}{l}\text { Gestão e políticas } \\
\text { públicas }\end{array}$ & $\begin{array}{l}\text { Gestão de riscos, planos de emergência e as relações das } \\
\text { políticas públicas }\end{array}$ & 6 \\
\hline Vulnerabilidade & Vulnerabilidade socioambiental & 5 \\
\hline Prevenção & $\begin{array}{l}\text { Prevenção de riscos de desastres, ações que buscam evitar que o } \\
\text { evento ocorra }\end{array}$ & 4 \\
\hline Mitigação & $\begin{array}{l}\text { Mitigação de acidentes e desastres, redução dos impactos do } \\
\text { evento }\end{array}$ & 3 \\
\hline Mudanças climáticas & Mudanças climáticas & 2 \\
\hline Formação & $\begin{array}{l}\text { Formação de moradores de áreas de riscos, técnicos do poder } \\
\text { público e professores }\end{array}$ & 2 \\
\hline Comunicação & Construção do discurso e formas simbólicas da mídia & 2 \\
\hline Psicossocial & Abordagem psicossocial com atores sociais em situação de risco & 12 \\
\hline Outros & Agrupamento de temas com apenas um trabalho & 2 \\
\hline
\end{tabular}

Quadro 1 - Frequência de ocorrência das categorias temáticas presentes nas pesquisas encontradas nas seis plataformas consultadas até março de $2019(\mathrm{~N}=53)$

Fonte: elaborada pelas autoras

Para fins de discussão e análise, nesta seção são apresentadas, com maiores detalhes, algumas temáticas, especificamente: Percepção, Ensino e currículo e Gestão de políticas públicas, por terem sido abordadas pelo maior número de publicações:

- Percepção: essa categoria incluiu trabalhos de avaliação da percepção de jovens afetados por enchentes de escolas públicas de União dos Palmares/AL e que discutiram o papel da educação ambiental no desenvolvimento da percepção desses riscos e na prevenção de acidentes. Mendonça e Gullo (2017) enfatizam que, sem a compreensão da percepção de risco da comunidade, as políticas públicas em RRD têm poucas chances de serem eficientes, por desconsiderarem essa perspectiva que agrega com as visões, conhecimentos, valores, crenças e sentimentos.

- Ensino e currículo: os trabalhos analisaram programas e projetos de educação ambiental de MG, RJ, PE e SP, como a influência do contexto local de riscos nas abordagens de educação ambiental de escolas situadas na área de influência direta do Polo Industrial de Campos Elíseos, em Duque de Caxias/RJ, e como os riscos ambientais são trabalhados no currículo escolar de uma escola de Belo Horizonte/MG. Essa última pesquisa está alinhada com as Diretrizes Curriculares Nacionais para a Educação Ambiental, pois considera que o planejamento curricular e a gestão da unidade de ensino devem contribuir para o "[...] estabelecimento das relações entre as mudanças do clima e o atual modelo de produção, consumo, organização social, visando à prevenção de desastres ambientais e à proteção das comunidades" (BRASIL, 2014, p. 53).

- Gestão e políticas públicas: as publicações agrupadas nessa categoria temática trataram do gerenciamento de riscos ambientais e de políticas públicas direcionadas à mitigação de riscos e à prevenção de desastres. O trabalho de Santos (2012) analisou como o Sistema de Gestão de Risco e a Política Pública de Assistência Social vêm sendo executados no município de Blumenau/SC. A autora também descreve a articulação 
realizada pelos afetados de desastres para a criação do MAD Blumenau - Movimento dos Atingidos pelos Desastres, uma organização formada em 2008 que busca garantir a participação dos atingidos nos processos de construção das ações governamentais.

\subsection{Algumas lacunas e oportunidades de pesquisas}

As lacunas apresentadas a seguir estão relacionadas ao reduzido número de publicações relacionadas às temáticas estabelecidas na Tabela 3, focadas na Formação, Comunicação, Participação e Fatores geradores de riscos.

\subsubsection{Formação}

O Marco de Sendai ${ }^{5}$ e a Política Nacional de Proteção e Defesa Civil (UNISDR, 2015b; BRASIL, 2012) estabelecem como prioridade a formação em RRD para a sociedade civil, técnicos dos órgãos públicos e do setor privado, com compartilhamento de experiências, lições aprendidas e boas práticas. Entretanto, foram encontrados apenas dois trabalhos na categoria Formação, dentre todos os trabalhos analisados. Um deles analisou o papel dos cursos de educação não formal voltado para moradores e técnicos sobre prevenção e mitigação de acidentes e desastres em áreas de risco a movimentos de massa oferecidos pela Prefeitura do Município de São Paulo em 2012 e 2013. A autora destaca que para o propósito das formações contribuírem na prevenção e mitigação de acidentes e desastres é necessário sua integração ao gerenciamento participativo de risco das comunidades (GOTO, 2014).

É importante ressaltar a necessidade de realização de pesquisas avaliativas voltadas aos processos formativos de RRD de públicos citados anteriormente, como também de agentes da Defesa Civil, estudantes de graduação e pós-graduação e de professores e professoras das escolas localizadas em áreas de risco. Além disso, é de extrema relevância que essas pesquisas relatem desde suas concepções, processos metodológicos e resultados que identifiquem boas práticas até os impactos efetivos na gestão de riscos.

\subsubsection{Comunicação}

O Sistema de Gestão de Riscos de Desastres centrado nas pessoas é composto por quatro eixos: 1) conhecimento dos riscos; 2) monitoramento e alerta; 3) comunicação; e 4) capacidade de resposta (MARCHEZINI, et al., 2017b). O terceiro eixo, comunicação, visa informar atores sociais que se encontram em possíveis riscos, mas apenas dois (2) trabalhos foram classificados na categoria Comunicação. O artigo de Teixeira (2012) analisou os desastres ambientais a partir do olhar midiático, elucidando como a mídia narra e representa esses imaginários por meio do discurso do jornal Zero Hora e das capas de revistas Época e Veja. A análise mostra como as formas simbólicas são selecionadas e empregadas assim como o discurso apoia-se em valores e interesses que permeiam essas mídias.

\subsubsection{Participação}

Para a participação da população na redução de risco de desastres são necessários espaços de diálogo e trabalho colaborativo com mecanismos transparentes para tomada local de decisões e formulação de políticas (GNDR, 2013). Apenas um trabalho abordou o tema

5 Marco de Sendai para a Redução do Risco de Desastres (2015-2030) é um acordo internacional, elaborado em 2015, durante a $3^{\text {a }}$ Conferência Mundial da ONU na cidade de Sendai, Japão. É o resultado de consultas e negociações intergovernamentais realizadas entre 2012 a 2015. 
Participação e foi agrupado na categoria Outros. Olivato (2013) descreve que a população local não participou do processo de gestão de riscos ambientais na bacia hidrográfica do rio Indaiá, Ubatuba/SP, fato demonstrado pela falta de informações de grande parte dos entrevistados sobre os instrumentos de gestão e o mapeamento técnico existente. Esse cenário reforça a necessidade de mais pesquisas que abordem e identifiquem quais são as formas de participação da sociedade na gestão de riscos no Brasil e, também, como estimular e qualificar essa participação.

\subsubsection{Fatores geradores dos riscos e complexidade}

Fatores geradores dos riscos também foi incluído na categoria Outros, já que apenas o trabalho de Bauer (2016) trouxe essa abordagem. A autora relata que identificou nos discursos dos atores sociais que as mudanças climáticas, os aspectos geográficos e a ocupação do solo são entendidos como causas geradoras de riscos de desastres na região do Vale do Itajaí/SC.

Oliver-Smith et al. (2017, p. 110) elencam dezesseis fatores de riscos e descrevem que as "causas básicas dos desastres estão profundamente inseridas nas escolhas e valores econômicos e socioculturais dominantes [...]". Associando-se à necessidade de aprender fatos novos e complexos por meio da perspectiva da complexidade nos processos educativos, com a construção de uma nova racionalidade, focada na emancipação que permita novas formas de reapropriação do mundo e de convivência com os outros, com a sustentabilidade e justiça (LEFF, 2009).

Sulaiman (2018) menciona a necessidade de a sociedade ter o entendimento desses fatores geradores de riscos para a prevenção dos desastres e que essa abordagem não tem sido frequente nas práticas educativas no Brasil, permanecendo, ainda, um enfoque informativo e procedimental.

Essa última lacuna reforça a interface com a perspectiva da complexidade e a corrente de educação ambiental crítica quanto à promoção e à desconstrução da realidade socioambiental em busca de diálogos e transformações das causas dos problemas (SAUVÉ, 2010) que contribuem para os cenários de desigualdades sociais e de urbanização em áreas de riscos e, também, com os processos econômicos, históricos e políticos.

\section{Considerações finais}

Os resultados da presente pesquisa do estado da arte seguem algumas tendências encontradas em outros estudos similares. A maioria das produções foi produzida em instituições públicas, localizadas na região Sudeste. Sendo assim, consideramos que há de se ampliar a abrangência e a divulgação desses trabalhos, pois existe grande potencial no desenvolvimento de futuras pesquisas em diversos contextos regionais.

Riscos e desastres são conceitos polissêmicos e a presença de publicações em todas as seis combinações de descritores utilizados reafirmam as múltiplas abordagens que a temática possibilita, ao mesmo tempo em que reforça que a RRD, enquanto novo campo de conhecimento, está em processo de construção e abre a discussão da necessidade de definição dos riscos e desastres adotados nessas pesquisas, sendo que a falta de explicitação das bases epistemológicas envolvidas pode levar a interpretações equivocadas dos resultados.

A pluralidade de conceitos também foi encontrada nos principais temas em que os descritores foram abordados. Porém, a existência de um reduzido número de pesquisas implicou na identificação de algumas lacunas: i) temáticas: formação de atores sociais em RRD; comunicação no sistema de alerta centrado nas pessoas; participação da população no gerenciamento de riscos de desastres; e fatores geradores de risco de desastres; ii) ameaças: inexistência de investigações que associaram a seca com os termos riscos e desastres. Sendo 
assim, os resultados deste artigo podem subsidiar futuros caminhos da produção acadêmica no país, com novas investigações, de média e longa duração, para contribuir nessa nova área da redução de riscos de desastres articulada ao campo da educação ambiental.

Com a perspectiva de aumento e intensificação de desastres no Brasil, associada aos milhões de brasileiros em áreas de riscos em todas as regiões, torna-se essencial a promoção de formações, eventos e espaços de diálogos que articulem e contribuam com a realização de novas pesquisas de educação ambiental e os riscos e desastres regionalizados.

Dessa forma, salientamos que avanços nas pesquisas sobre educação ambiental e RRD são necessários para o fortalecimento desse campo de atuação e, também, para criar uma cultura de prevenção de riscos de desastres, de segurança e resiliência em todos os níveis no Brasil.

\section{Agradecimentos}

As autoras agradecem ao Conselho Nacional de Desenvolvimento Científico e Tecnológico (CNPq) pelas bolsas concedidas, e ao Grupo de Pesquisa em Educação Ambiental e Formação de Educadores (GPEAFE) da Universidade de São Paulo.

\section{Referências}

BARDIN, L. Análise de conteúdo. São Paulo: Edições 70, 2016.

BAUER, V.C. As concepções de um grupo de atores sociais sobre mudança climática, riscos de desastres ambientais e vulnerabilidade no Vale do Itajaí-SC. 2016. 114f. Dissertação (Mestrado em Educação) - Universidade do Vale do Itajaí, Itajaí, 2016.

BECK, U. Sociedade de Risco: Rumo a uma outra modernidade. São Paulo: Editora 34, 2010.

BRASIL. Lei $N^{o}$ 12.608, de 10 de Abril de 2012. Institui a Política Nacional de Proteção e Defesa Civil - PNPDEC. Brasília: Casa Civil, 2012. Disponível em: <http://www.planalto.gov.br/ccivil_03/_Ato2011-2014/2012/Lei/L12608.htm>. Acesso em 12 mai. 2019.

BRASIL. Diretrizes Curriculares Nacionais para a Educação Ambiental. In: BRASIL. Ministério do Meio Ambiente. Ministério da Educação. Educação Ambiental Por um Brasil Sustentável: ProNEA, Marcos Legais \& Normativos. Brasília: MMA/MEC, 2014. p.47-55.

CARPI Jr., S. Identificação de riscos ambientais e proteção da água: uma aproximação necessária. In: LIMA-GUIMARÃES, S.T.; CARPI JR., S.; BERRÍOS, M.B.R.; TAVARES, A.C. (Orgs.). Gestão de áreas de riscos e desastres ambientais. Rio Claro: IGCE/UNESP/RIO CLARO, 2012. p.32-59.

CARVALHO, I. C. M. Educação ambiental crítica: nomes e endereçamentos da educação In: LAYRARGUES, P. P. (Coord.) Identidades da Educação ambiental brasileira. Brasília: Ministério do Meio Ambiente, 2004. p.13-24.

CARVALHO, I.C.M.; FARIAS, C.R.O. Um balanço da produção científica em educação ambiental de 2001 a 2009 (ANPEd, ANPPAS e EPEA). Revista Brasileira de Educação, Rio de Janeiro, v.16, n.46, p.119-267, jan-abr. 2011.

COORDENAÇÃO DE APERFEIÇOAMENTO DE PESSOAL DE NÍVEL SUPERIOR (CAPES 2017) Avaliação da CAPES aponta crescimento da pós-graduação brasileira. Disponível em: 
$<$ https://www.capes.gov.br/sala-de-imprensa/noticias/8558-avaliacao-da-capes-aponta-crescimentoda-pos-graduacao-brasileira>. Acesso em 12 abr. 2019.

COUTINHO, S.; RAMOS, R.R. Desafio presente: mudanças climáticas e desastres naturais. In: SULAIMAN, S.N.; JACOBI, P.R. (Orgs.). Melhor prevenir: olhares e saberes para a redução de risco de desastre. São Paulo: IEE-USP, 2018. p.40-48.

CUNHA, A.P.; MARENGO, J.A.; ALVALA, R.C.; DEUSDARA-LEAL, K.R.; CUARTAS, L.A.; SELUCHI, M.; ZERI, M.; RIBEIRO-NETO, G.; BRODEL, E.; CUNNINGHAM, C.; COSTA, L.; MORAES, O.L.L. Secas e seus Impactos no Brasil 2018. Cemaden, São José dos Campos, s/v, n.1, p.19, 2019.

DA SILVA-ROSA, T.; MENDONÇA, M.B.; MONTEIRO, T.G.; SOUZA, R.M.; LUCENA, R. A educação ambiental como estratégia para a redução de riscos socioambientais. Ambiente \& Sociedade, São Paulo, v.XVIII, n.3, p.211-230, 2015.

FERREIRA, N. S. A. As pesquisas denominadas "estado da arte". Educação \& Sociedade, Campinas, v. 23, n. 79, p. 257-272, 2002.

FRANCO, M.L.P.B. Análise de conteúdo. Brasília: Plano Editora, 2003.

GLOBAL NETWORK OF CIVIL SOCIETY ORGANISATIONS FOR DISASTER REDUCTION (GNDR). Views from the Frontline 2013. Disponível em: 〈https://gndr.org/programmes/vfl/vfl-2013〉. Acesso em: 11 mai. 2019.

GOTO, E.A. Cursos de educação não formal voltados para moradores de áreas de risco e técnicos da prefeitura: uma análise do seu papel. 2014. 134f. Dissertação (Mestrado em Ensino e História de Ciências da Terra) - Universidade Estadual de Campinas, Campinas, 2014.

INTERGOVERNMENTAL PANEL ON CLIMATE CHANGE (IPCC) Climate Change 2014: Synthesis Report. Contribution of Working Groups I, II and III to the Fifth Assessment Report of the Intergovernmental Panel on Climate Change. PACHAURI, R.K.; MEYER L.A. (Eds.). IPCC, Geneva, Switzerland, 2014.

INTERNATIONAL DISASTER DATABASE (EM-DAT) - Country Profile. Disponível em: <www.emdat.be>. Acesso em: 11 jan. 2019.

INSTITUTO BRASILEIRO DE GEOGRAFIA E ESTATÍSTICA (IBGE); CENTRO NACIONAL DE MONITORAMENTO E ALERTAS DE DESASTRES NATURAIS (CEMADEN). População em áreas de risco no Brasil. Rio de Janeiro: IBGE; CEMADEN, 2018.

INSTITUTO BRASILEIRO DE GEOGRAFIA E ESTATÍSTICA (IBGE) Perfil dos municípios brasileiros: 2017/IBGE Coordenação de População e Indicadores Sociais. Rio de Janeiro: IBGE, 2017.

JACOBI, P.R. Mudanças climáticas e ensino superior: a combinação entre pesquisa e educação. Educar em Revista, Curitiba, Edição Especial, n.3, p.57-72, 2014.

LEFF, E. Complexidade, racionalidade ambiental e diálogo de saberes. Educação \& Realidade, Porto Alegre, v.34, n.3, p.17-24, 2009.

LOUREIRO, C. F. B. Educação ambiental e gestão participativa na explicitação e resolução de conflitos. Gestão em Ação, Salvador, v.7, n.1, p.37-50, 2004. 
MARCHEZINI, V.; WISNER, B.; LONDE, L.R.; SATO, S.M. Por que vulnerabilidade? In: MARCHEZINI, V.; WISNER, B.; LONDE, L.R.; SATO, S.M. (Orgs.) Reduction of vulnerability to disasters: from knowledge to action. São Carlos: RiMa Editora, 2017a. p. 45-53.

MARCHEZINI, V.; TRAJBER, R.; OLIVATO, D.; MUÑOZ, V.A.; PEREIRA, F.O.; LUZ, A.E.O. Participatory Early Warning Systems: Youth, Citizen Science, and Intergenerational Dialogues on Disaster Risk Reduction in Brazil. Int J Disaster Risk Sci, n.8, p.390-401, 2017 b.

MARCHEZINI, V.; MUÑOZ, V. A.; TRAJBER, R. Vulnerabilidade Escolar frente a Desastres no Brasil. Territorium, v.25, n.II, p.161-177, 2018a.

MARCHEZINI, V.; HORITA, F.E.A.; MATSUO, P.M.; TRAJBER, R.; TREJO-RANGEL, M.A.; OLIVATO, D. A Review of Studies on Participatory Early Warning Systems (P-EWS): Pathways to Support Citizen Science Initiatives. Frontiers In Earth Science, Lausanne, v.6, n.184, p.1-18, 2018 b.

MARENGO, J. A. Mudanças Climáticas, Condições Meteorológicas Extremas e Eventos Climáticos no Brasil. In: FBDS (Org.) Mudanças Climáticas Eventos Extremos no Brasil. Rio de Janeiro: FDBS \& LLOYD’S, 2010. p.05-19.

MAZZUCATO, E.; BACCI, D. C. Estado da Arte e Contribuição das Geociências para Educação Ambiental. Pesquisa em Educação Ambiental, Rio Claro, v.12, n.2, p.141-161, fev. 2017.

MENDONÇA, M.B.; GULLO, F.T. Percepções de risco associado a deslizamentos em Angra dos Reis, Rio de Janeiro. In: MARCHEZINI, V.; WISNER, B.; SAITO, S.M.; LONDE, L.R. (Eds.) Reduction of Vulnerability to Disasters: from Knowledge to Action. São Carlos: Rima Editora, 2017. p.477- 497.

MORIN, E. Introdução ao pensamento complexo. 5.ed. - Porto Alegre: Sulina, 2015.

MOURA, R.B. Angra dos Reis: Resiliência pós-desastres de 2009/2011. In: SULAIMAN, S.N.; JACOBI, P.R. (Orgs.). Melhor prevenir: olhares e saberes para a redução de risco de desastre. São Paulo: IEE-USP, 2018. p.60-71.

OLIVATO, D. Análise da participação social no contexto da gestão de riscos ambientais na bacia hidrográfica do rio Indaiá, Ubatuba-SP-Brasil. 2013. 292f. Tese (Doutorado em Geografia Física) Universidade de São Paulo, São Paulo, 2013.

OLIVER-SMITH, A.; ALCÁNTARA-AYALA, I.; BURTON, I.; LAVELL, A. A construção social do risco de desastres: em busca das causas básicas. In: MARCHEZINI, V.; WISNER, B.; SAITO, S.M.; LONDE, L.R. (Eds.) Reduction of Vulnerability to Disasters: from Knowledge to Action. São Carlos: Rima Editora, 2017. p.94-114.

ROMANOWSKI, J. P.; ENS, R. T. As pesquisas denominadas do tipo "estado da arte" em educação. Diálogo Educ., Curitiba, v.6, n.19, p.37-50, 2006.

SANTOS, R. Gestão de desastres e política de assistência social: estudo de caso de Blumenau/SC. 2012. 336f. Dissertação (Mestrado em Sociologia Política) - Universidade Federal de Santa Catarina, Florianópolis, 2012.

SAUVÉ, L. Educación científica y educación ambiental: un cruce fecundo. Enseñanza de las Ciencias. Barcelona, v.28, n. 1, p.05-18, 2010.

SELBY, D. KAGAWA, F. Disaster Risk Reduction in School Curricula: Case Studies from Thirty Countries. Genebra: UNESCO/UNICEF, 2012. 
SILVA, R. L. F.; CAMPINA, N. N. Concepções de educação ambiental na mídia e em práticas escolares: contribuições de uma tipologia. Pesquisa em Educação Ambiental, Rio Claro, v.6, n. 1, p.29-46, jan-jun. 2011.

SULAIMAN, S.N. Ação e reflexão: Educar para uma cultura preventiva. In: SULAIMAN, S.N.; JACOBI, P.R. (orgs.). Melhor prevenir: olhares e saberes para a redução de risco de desastre. São Paulo: IEE-USP, 2018. p.22-29.

TEIXEIRA, D.V. Os desastres ambientais a partir do olhar midiático. Revista Eletrônica do Mestrado em Educação Ambiental, Rio Grande, v. 28, p.1517-1256, jan-jun. 2012.

TOMINAGA, L.K.; SANTORO, J.; AMARAL, R. (Orgs.). Desastres naturais: conhecer para prevenir. 3 ed. São Paulo: Instituto Geológico, 2015.

TRAJBER, R.; OLIVATO, D.; MARCHEZINI, V. Conceitos e termos para a gestão de riscos de desastres na educação. Projeto Cemaden Educação. São José dos Campos: CEMADEN, 2015. Disponível em: <http://educacao.cemaden.gov.br/site/mediaLibrary/MTAwMDAwMDAwMTg=>. Acesso em: 22 abr. 2019.

UNITED NATIONS CHILDREN FUND (UNICEF). UNITED NATIONS OFFICE FOR DISASTER RISK REDUCTION (UNISDR) Children and disasters: Building resilience through education. Genebra: UNICEF/UNISDR, 2011.

UNITED NATIONS OFFICE FOR DISASTER RISK REDUCTION (UNISDR). Terminología sobre reducción del riesgo de desastres. Genebra: UNISDR, 2009.

UNITED NATIONS OFFICE FOR DISASTER RISK REDUCTION (UNISDR). Global Assessment Report on Disaster Reports on Disaster Risk Reduction (GAR), Risk Reduction Making Development Sustainable: the future of disaster risk management. Geneva: UNISDR, 2015a.

UNITED NATIONS OFFICE FOR DISASTER RISK REDUCTION (UNISDR). Marco de Sendai para a Redução do Risco de Desastres 2015-2030. Sendai: UNISDR, 2015 b.

VIEIRA, R.; JANSEN, G.R. Blumenau: contribuições para a gestão de risco no Brasil. In: SULAIMAN, S.N.; JACOBI, P.R. (Orgs.). Melhor prevenir: olhares e saberes para a redução de risco de desastre. São Paulo: IEE-USP, 2018. p.106-110.

WISNER, B.; BLAIKIE, P.; CANNON, T.; DAVIS, I. At Risk: natural hazards, people's vulnerability and disasters. 2 ed. London: Routledge, 2003. 